\title{
Early progression of pulmonary hypertension in the monocrotaline model in males is associated with increased lung permeability
}

Olga Rafikova, Joel James, Cody A. Eccles, Sergey Kurdyukov, Maki Niihori, Mathews Valuparampil Varghese and Ruslan Rafikov*

\begin{abstract}
Background: The mechanisms involved in pulmonary hypertension $(\mathrm{PH})$ development in patients and pre-clinical models are poorly understood. PH has a well-established sex dimorphism in patients with increased frequency of $\mathrm{PH}$ in females, and more severe disease with poor survival prognosis in males. Previously, we found that heme signaling plays an essential role in the development phase of the Sugen/Hypoxia (SU/Hx) model. This study is focused on the elucidation of sex differences in mechanisms of $\mathrm{PH}$ development related to heme action at the early stage of the monocrotaline (MCT) PH model.
\end{abstract}

Methods: Rats received MCT injection (60 mg/kg, i.p.) and followed for 14 days to investigate early disease changes. Hemodynamic parameters were recorded at the end of the study; plasma, lung homogenates, and nuclear fractions were used for the evaluation of protein levels.

Results: Our data indicate that on day 14 , rats did not show any significant increase in the Fulton index due to the early disease phase. However, the right ventricular systolic pressure was significantly increased in male rats, while female rats showed only a trend. Interestingly, only males demonstrated an increased lung-to-bodyweight ratio that indicated lung edema. Indeed, lung histology confirmed severe perivascular edema in males. Previously, we have reported that the increased perivascular edema in SU/Hx model correlated with intravascular hemolysis and activated heme signaling. Here, we found that elevated free hemoglobin levels and perivascular edema were increased, specifically in males showing more rapid progress of $\mathrm{PH}$. A high level of heme carrier protein 1 (HCP-1), which is involved in heme uptake from the bloodstream into the cells, was also found elevated in the lungs of males. The upregulation of heme oxygenase in males indicated increased intracellular heme catabolism. Increased heme signaling resulted in the activation of heme-mediated barrier-disruptive mechanisms. Thus, hemolysis in males can be responsible for increased permeability of the lungs and early disease development.

Conclusions: Our study indicates the importance of barrier-disruptive mechanisms as an earlier event in the induction of pulmonary hypertension. Importantly, males are more susceptible to hemolysis and develop PH earlier than females.

Keywords: Pulmonary hypertension, Heme signaling, Lung permeability, Sex difference, Endothelial barrier function

\footnotetext{
* Correspondence: ruslanrafikov@email.arizona.edu

Division of Endocrinology, Department of Medicine, University of Arizona,

Tucson, AZ 85721, USA
}

(c) The Author(s). 2020 Open Access This article is licensed under a Creative Commons Attribution 4.0 International License, which permits use, sharing, adaptation, distribution and reproduction in any medium or format, as long as you give appropriate credit to the original author(s) and the source, provide a link to the Creative Commons licence, and indicate if changes were made. The images or other third party material in this article are included in the article's Creative Commons licence, unless indicated otherwise in a credit line to the material. If material is not included in the article's Creative Commons licence and your intended use is not permitted by statutory regulation or exceeds the permitted use, you will need to obtain permission directly from the copyright holder. To view a copy of this licence, visit http://creativecommons.org/licenses/by/4.0/ The Creative Commons Public Domain Dedication waiver (http://creativecommons.org/publicdomain/zero/1.0/) applies to the data made available in this article, unless otherwise stated in a credit line to the data. 


\section{Background}

Pulmonary hypertension $(\mathrm{PH})$ affects vascular circulation in the lungs. Pulmonary vasculature becomes narrowed due to vasoconstriction, proliferation, fibrosis, or thrombosis, leading to increased pressure in the pulmonary circulation and increased workload for the right heart [1-3]. Patients with PH die due to the right heart failure. However, the main problem stems from vascular wall cells' proliferation, which includes endothelial [4-6], smooth muscle cells $[7,8]$, and fibroblasts $[9,10]$. There are several animal models available to study pulmonary hypertension, including the well-established monocrotaline (MCT) $[11,12]$ and Su5416/hypoxia (SU/Hx) $[13,14]$ models. The MCT model involves a one-time injection of the plant toxin, monocrotaline that metabolizes in the liver, and produces the monocrotaline pyrrole. MCT pyrrole induces endothelial damage via induction of oxidative stress. This oxidative stress occurs due to the binding of monocrotaline pyrrole to free cysteine residues or by direct inactivation of catalase [15]. SU/Hx model reproduces the main characteristics of human-like pulmonary hypertension with high pulmonary pressure, irreversible proliferative remodeling in the pulmonary artery with plexiform lesions formation, and right heart hypertrophy. However, the mechanism of pulmonary hypertension induction is still in debate. As a VEGFR2 blocker, SU5416 induces initial damage to the endothelial cells by preventing their growth and by induction of apoptosis [13, 14]. Thus, endothelial damage could be a common mechanism of disease initiation in both animal models.

Pulmonary hypertension is a complex disease, which includes activation and inhibition of the same pathways throughout the course of the disease. For example, apoptosis initially activated in $\mathrm{PH}$ is later replaced by the uncontrolled growth of the cells in the vascular wall [14]. Using the MCT model at the early stage of the disease, 14 days after MCT injection, we previously demonstrated that activation of many important pathways involved in $\mathrm{PH}$ pathobiology precedes any histological and pathophysiological changes [16]. Thus, we found that at the beginning of the disease, cells in the lungs significantly change their metabolism, leading to alterations in arginine/nitric oxide pathway, glycolysis, prostaglandin synthesis, glutathione balance, extracellular matrix formation, and activity of inflammatory pathways. Therefore, focusing on early events in the pulmonary circulation can shed light on important mechanisms leading to pathological changes in $\mathrm{PH}$ [2].

Recently, we have reported that patients with $\mathrm{PH}$ have increased levels of circulating free hemoglobin $(\mathrm{Hb})$, a marker of hemolysis, which strongly correlated with the clinical characteristics of $\mathrm{PH}$ progression [17]. In the $\mathrm{SU} / \mathrm{Hx}$ model, hemolysis was associated with an increased heme-mediated endothelial barrier dysfunction and formation of perivascular edema. These changes were especially evident in the early stages of the disease. Therefore, in the present work, we aimed to investigate the early stage of the MCT model for heme-mediated pathways as well as a possible contribution of sex, by separate analysis of male and female rats.

\section{Methods}

\section{Animal model}

A total of 24 Sprague Dawley rats (12 weeks old), 12 male and 12 female animals, were used in this study ( $N$ $=6$ per group). A total of 6 male and 6 female animals were injected intraperitoneally (i.p.) with monocrotaline (MCT) at $60 \mathrm{mg} / \mathrm{kg}$ at day 1 of the experiment. Six control animals were injected i.p. with monocrotaline vehicle. Animals under study were housed at the University of Arizona's Animal Care Facility until day 14 of the experiment.

Animals were housed at an ambient $22^{\circ} \mathrm{C}$ with a 12 -h light/dark cycle. All animals received standardized rodent food and water ad libitum. The handling procedures and experimental design were approved by the University of Arizona Institutional Animal Care and Use Committee.

\section{Hemodynamic measurements and sample collection}

Fourteen days post-MCT/vehicle treatment; animals were anesthetized with Inactin (Sigma-Aldrich, T133) at the dosage of $100 \mathrm{mg} / \mathrm{kg}$, i.p. A customized pressure transducer catheter (SPR-513, Millar Instruments, Houston, TX) was inserted through the right jugular vein and advanced into the right ventricle (RV) to monitor right ventricular systolic pressure (RVSP) and right ventricular diastolic pressure (RVDP) as described previously [17]. Briefly, the pressure transducer catheter was connected to a Millar Transducer Control Unit TC-510 and PL3504 PowerLab 4/35 data acquisition system (AD Instruments, Colorado Springs, CO) to continuously record RV pressure under small animal ventilator system (Harvard Rodent Ventilator-683; Harvard Apparatus, South Natick, MA). The thorax was opened, $4-5 \mathrm{~mL}$ of whole blood was collected into a heparinized syringe through the right ventricle (RV), centrifuged at $1000 \mathrm{~g}$ for $2 \mathrm{~min}$; separated plasma was aspirated and snapfrozen in liquid nitrogen for later analysis. Next, the lungs were flushed with $0.9 \%$ sodium chloride through the RV. Heart and lungs were subsequently collected from the animals; the RV free wall was separated from the left ventricle (LV) and the septum (S). The Fulton index $(\mathrm{RV} / \mathrm{LV}+\mathrm{S}$ ratio) was assessed as a parameter of RV hypertrophy. The total wet lung weight was measured and normalized to the bodyweight of the animal. The left lung was fixed in formalin and embedded in paraffin for histological examination. The right lung was 
snap-frozen in liquid nitrogen and stored at $-80^{\circ} \mathrm{C}$ for further biochemical studies.

\section{Cell-free hemoglobin measurement}

Plasma analysis of cell-free hemoglobin was done utilizing gel electrophoresis and subsequent excitation at 488 $\mathrm{nm}$, as described previously [17]. Briefly, $1 \mathrm{uL}$ of plasma was diluted 10 times with PBS, solvated with $6 \times$ nonreducing Laemmli sample buffer (Boston Bioproducts Inc., Ashland, MA), loaded into 4-20\% SDS-PAGE Mini-PROTEAN TGX ${ }^{\text {sm }}$ gels (Bio-Rad Laboratories Inc., Hercules, CA) and separated by electrophoresis. The auto-fluorescent bands were visualized by $488 \mathrm{~nm}$ light emitted in a ChemiDoc ${ }^{\mathrm{m}}$ MP Imaging System (Bio-Rad Laboratories Inc., Hercules, CA) and analyzed using Image Lab $^{\text {Tx }}$ software.

\section{Western blot analysis}

Total lung protein was analyzed as previously described [17]. Briefly, the frozen lung was lysed in a permeabilization buffer with protease and phosphatase inhibitor cocktail (ThermoScientific) and mechanically homogenized using a Fisher Homogenizer 850. The samples were centrifuged at $10,000 \mathrm{~g}$ for $10 \mathrm{~min}$, and the supernatant was collected. Protein quantification was done utilizing a BCA protein assay kit (Pierce ${ }^{\mathrm{m}}$, Rockford, IL). A total of $20-40 \mu \mathrm{g}$ of protein were incubated with $6 \times$ Laemmli sample buffer (Boston Bioproducts Inc., Ashland, MA) for $5 \mathrm{~min}$ at $95^{\circ} \mathrm{C}$, and loaded into 4-20\% SDS-PAGE Mini-PROTEAN TGX Stain-Free ${ }^{\text {Tn }}$ gels (Bio-Rad Laboratories Inc., Hercules, CA) and separated by electrophoresis. Protein was transferred from the gel onto PVDF membranes using the Trans-Blot Turbo transferring system (Bio-Rad Laboratories Inc.) and blocked with $5 \%$ bovine serum albumin in Tris-buffered saline. Membranes were probed for HCP1 (B-4) (Santa Cruz, sc-393460), Heme Oxygenase 1 (A-3) (Santa Cruz, sc-136960), NFE2L2 (Cell Signaling, 12721S), NRF1 (Cell Signaling, 69432), Bach1 (Santa Cruz, sc-271211), HSP27 (G31) (Cell Signaling, 2402S), Rac1 (C-14) (Santa Cruz, sc217), P-MYPT1 (Thr696) (Cell Signaling, 5163S), MYPT1 (Cell Signaling, 2634S), P-MLC2 (Ser19) (Cell Signaling, 3671P), MLC2 (Cell Signaling, 3672S), Claudin-1 (Abcam, Ab140349), Claudin-5 (Abcam, Ab15106), ZO-1 (Cell Signaling, 5406S). Some membranes were re-probed for multiple proteins; probed membranes were stripped at room temperature for $10 \mathrm{~min}$ with membrane stripper (ThermoFisher). The protein loading was normalized per total sample protein using stain-free gels, as previously described [17]. This normalization is equal to housekeeping genes normalization and rigorously evaluated by the Bio-Rad company (http://www.bio-rad. com/en-us/applications-technologies/stain-free-imaging -technology?ID=NZ0G1815) and by our lab in comparison with beta-actin normalization.

\section{Lung tissue subcellular fractionation}

Subcellular protein fractionation was completed utilizing FractionPREP $^{\mathrm{mi}}$ Cell Fractionation Kit (BioVision Inc., Milpitas, Ca). The frozen lung tissue was processed as per the manufacturer's provided protocol. Subsequently, the nuclear fractions were quantified with the BCA protein assay kit (Pierce ${ }^{\mathrm{Tm}}$, Rockford, IL) and subjected to western blot analysis. Proteins were normalized on a stain-free total protein signal in the nuclear fraction.

\section{Histological analysis}

For the morphometric assessment of pulmonary vessels, $5-\mu \mathrm{m}$ tissue sections were dewaxed and stained with hematoxylin and eosin (H\&E) by HistoWiz Inc. (histowiz. com) using standard operating procedures and a fully automated workflow. The wall thickness of the pulmonary artery (PA) was measured using the publicly available software Fiji ImageJ (http://fiji.sc/Fiji; in the public domain) [18].

\section{Statistical analysis}

The means \pm SEM were calculated, and significance was determined by the two-way analysis of variance (ANOVA) or the unpaired $t$ test. The factors considered in the two-way ANOVA was the sex of the animal group and MCT treatment. For two-way ANOVA, Sidak's post hoc testing was also utilized. The unpaired $t$ test was carried out between the controls of the respective groups-male control vs male MCT and female control vs female MCT. A value of $P<0.05$ was considered significant. Statistical calculations were performed using the GraphPad Prism 8 software.

\section{Results}

Male rats are prone to early susceptibility of the disease

In this study, the MCT rat model of $\mathrm{PH}$ was used. To assess the developmental phase of the disease as previously confirmed [16], the rats were analyzed 14 days after MCT injection. The male and female sexes were examined separately. Right ventricle catheterization indicates that at this early stage, the increase in the right ventricular systolic pressure (RVSP) was evident only in the male group (Fig. 1a). Right ventricle hypertrophy (measured by Fulton index) showed only a mild trend of increase in both males and females without reaching statistical significance (Fig. 1b). Still, the heart function in males was altered, as indicated by $\mathrm{dP} / \mathrm{dt}$ values (Fig. 1c and d). Thus, there was a significant increase in RV contractility and a trend of increasing RV relaxation in males.

We have previously found significant lung edema occurring at the early stages of the SU/Hx model. In this study, we observed a significantly increased lung weight in MCT-treated males (Fig. 2a), whereas female rats did not show increased lung weight. Histological examination 

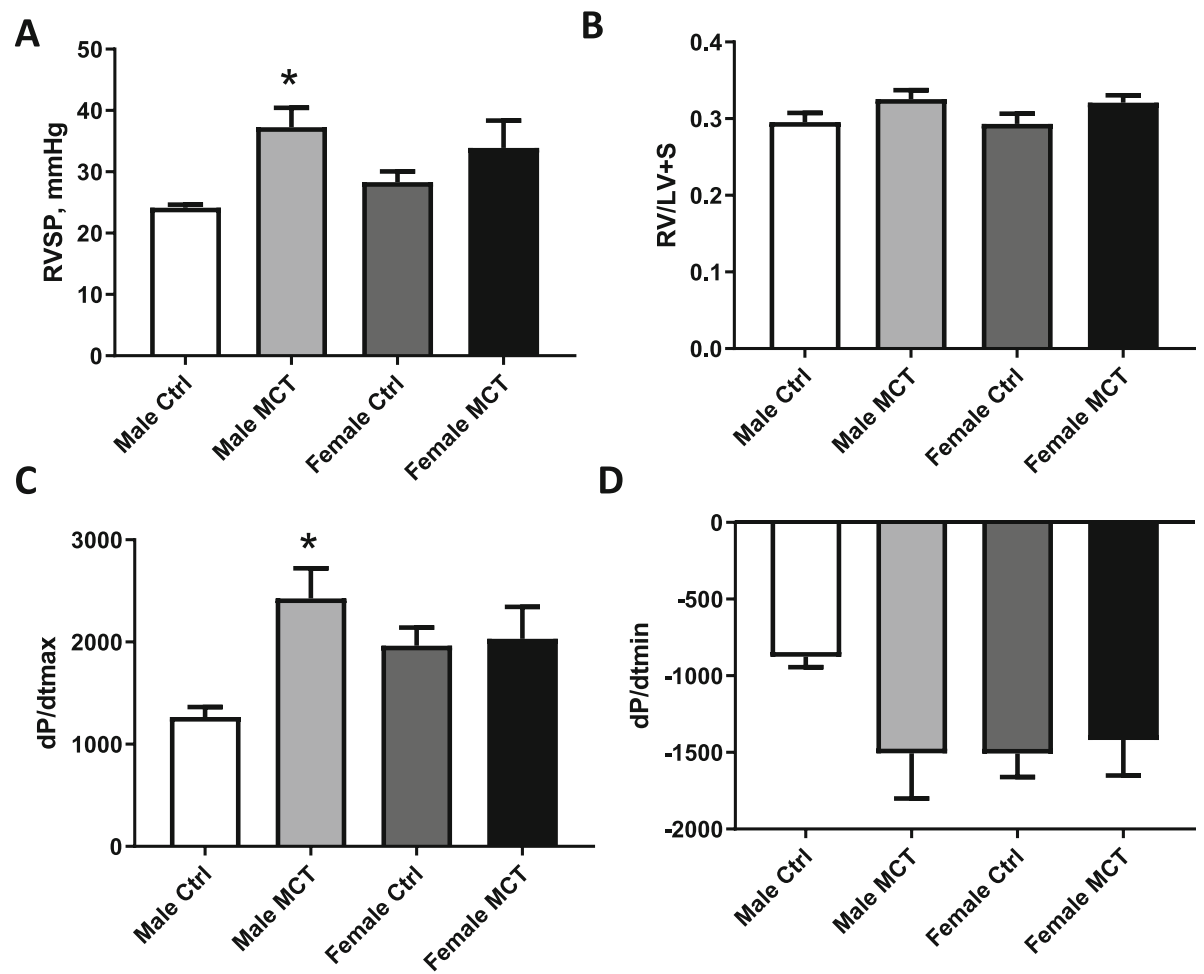

Fig. 1 Hemodynamic changes in 14 days MCT model. Right ventricular (RV) systolic pressure found to be elevated significantly only in males treated with MCT. a Fulton index at this early stage of PH was unchanged between groups. b Contractility of the right heart (dP/dtmax) was significantly reduced in males, but not in females. c Relaxation parameter (dP/dtmin) did not reach statistical significance in males and was unaffected in females. $\mathbf{d}$ Mean $\pm \mathrm{SEM}, N=6,{ }^{*} p<0.05$ vs control, two-way ANOVA

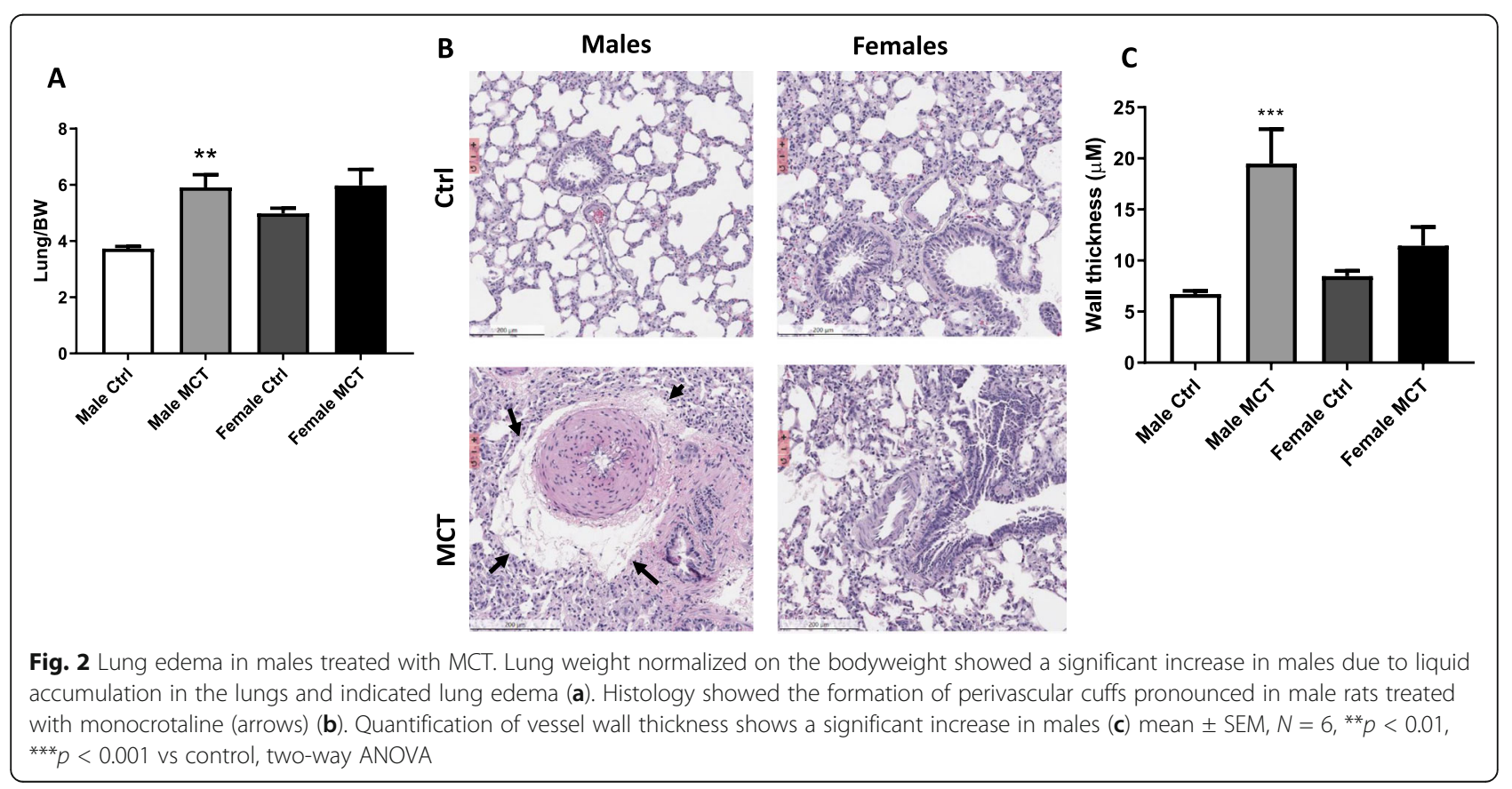


of lungs revealed the formation of perivascular edema and increased pulmonary artery wall thickness in males (Fig. 2b and c) that was consistent with the lung weight findings and vascular remodeling. Indeed, we have recently shown a direct correlation between the manifestation of disease and lung edema in both MCT and SU/Hx models [2].

\section{Male rats demonstrate increased hemolysis in the early MCT model}

Previously, we have reported that vascular permeability increases due to hemolysis, and free heme affect the endothelial barrier [17]. In order to evaluate hemolysis, we measured free hemoglobin levels in plasma. Our data indicate an increased level of free hemoglobin in males, whereas no changes were found in females (Fig. 3a). Heme uptake into vascular cells is regulated by heme carrier protein-1 (HCP1). We found that HCP-1 was upregulated only in males (Fig. $3 \mathrm{~b}$ ). Importantly, the heme oxygenase-1 (HO1), which is induced by increased heme concentration inside cells, was also found to be significantly upregulated in males (Fig. 3c). Thus, this confirms increased hemolysis and the entrance of free heme into the cells in males.

High levels of $\mathrm{HO} 1$ expression in males indicate a difference in transcriptional regulation between the sexes. To find out why males predominantly promote hememediated intracellular signaling, we tested the Nuclear factor erythroid 2-related factor 2 (NRF2) transcription factor (TF), which is linked with heme oxygenase and other antioxidant systems' upregulation. NRF2 works together with a repressor, BTB Domain, and CNC Homo$\log 1$ (BACH1). It has been previously shown that when heme binds to $\mathrm{BACH} 1$, it dissociates from the promoter region, which becomes available for NRF2 binding [19, 20]. Interestingly, we found that only males have an increased NRF2 expression that could be the primary mechanism of HO-1 upregulation (Fig. 4a) in males. The negative regulator of NRF2, BACH1, did not change in both males and females (Fig. 4b). Nuclear respiratory factor 1 (NRF1) is a TF, which upregulates in response to heme oxygenase and controls mitochondrial biogenesis. It was found to be increased in males (Fig. 4c). This result indicates that heme induces stress-related signaling and activates transcription factors only in males, leading to antioxidant response and mitochondrial biogenesis.

\section{Increased barrier-disruptive mechanisms in males in the early MCT model}

To determine whether free heme is involved in increased vascular permeability, we tested proteins involved in endothelial cell-to-cell adherence and barrier function. We found that HSP27, a heat shock family protein that is responsible for stabilization of actin and formation of stress fibers, was upregulated specifically in males (Fig. 5a). Subsequently, activation of cytoskeleton remodeling by myosin light chain (MLC) phosphorylation was found to be significantly increased in males (Fig. 5b). Conversely, the barrier protective mechanism that manifests via the stabilization of cortical actin filaments by Rac1 small GTPase [21]

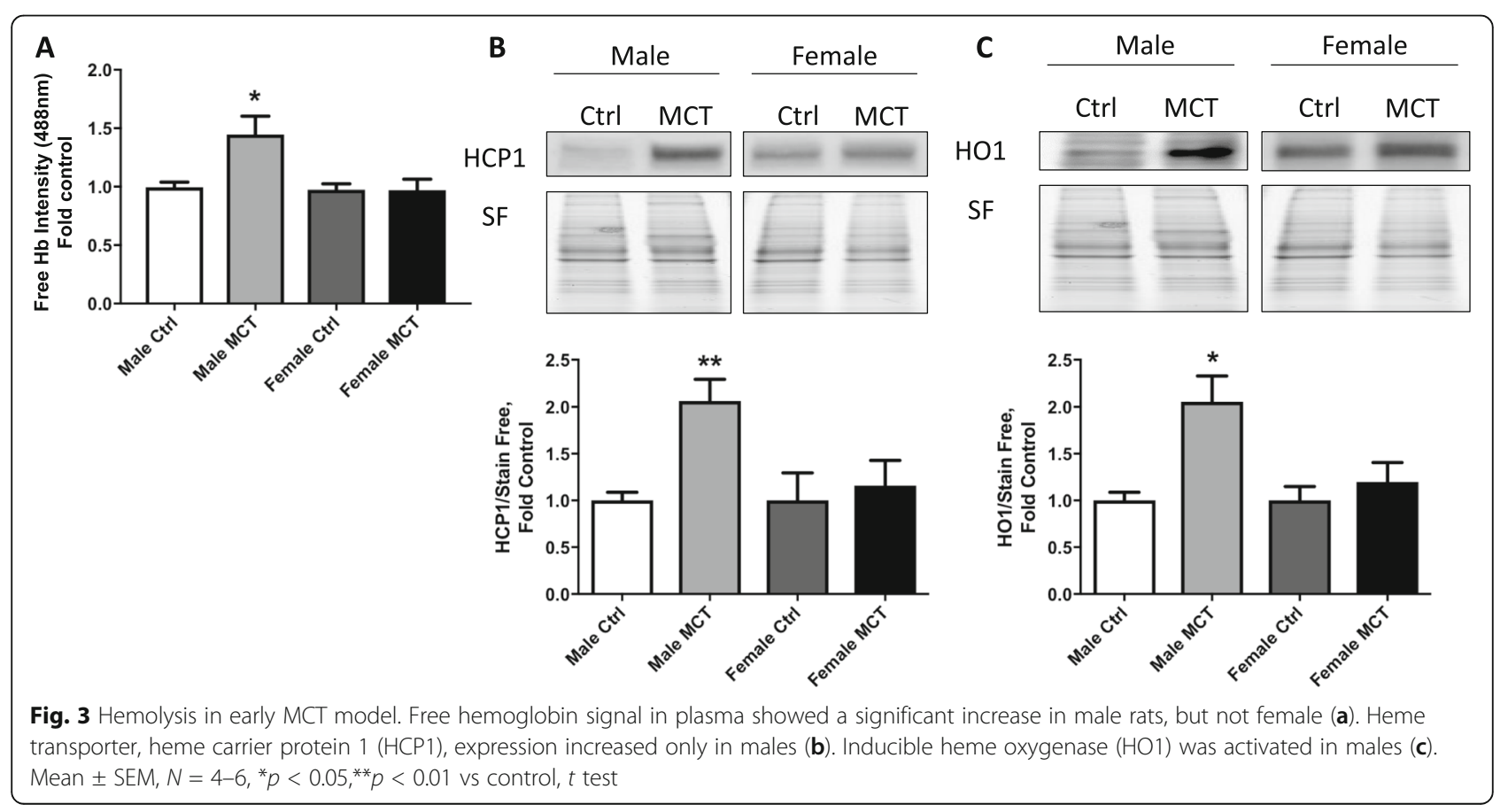



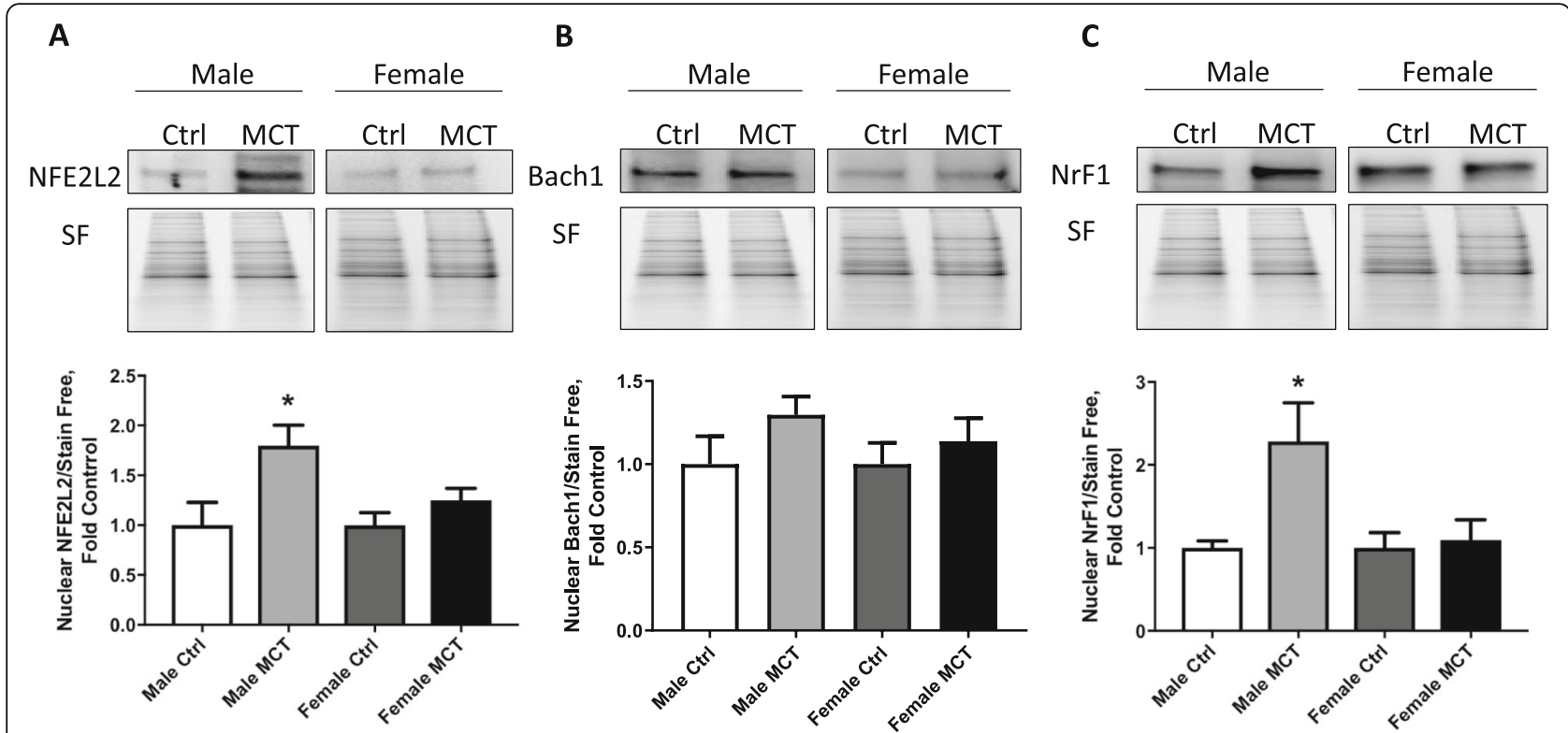

Fig. 4 Translocation of transcriptional factors (TF). Nuclear fractions that were isolated from lung tissue were used to screen the activation of different transcription factors. It was found that the factor responsible for heme-mediated response, NFE2L2 (or NRF2), was activated in males only (a). Transcription suppressor BACH1 was unchanged (b). Energy regulating TF, NRF1, was also activated in males (c). Nuclear proteins were normalized over their respective total proteins (stain-free). Mean $\pm \mathrm{SEM}, N=4,{ }^{*} p<0.05$ vs control, $t$ test

exhibited attenuation in males (Fig. 5c). The redistribution of actin from the plasma membrane into stress fibers is usually accompanied by a rearrangement of tight junctions (TJ), leading to decreased adherence between endothelial cells. We examined the main TJ proteins, such as Claudin-
$1 / 5$ and ZO-1. Our data indicate that TJ proteins were markedly reduced in males (Fig. 6a, b, c). This will result in the disruption of the endothelial barrier and fluid leakage from the bloodstream into the perivascular area, as we observed in male rats.

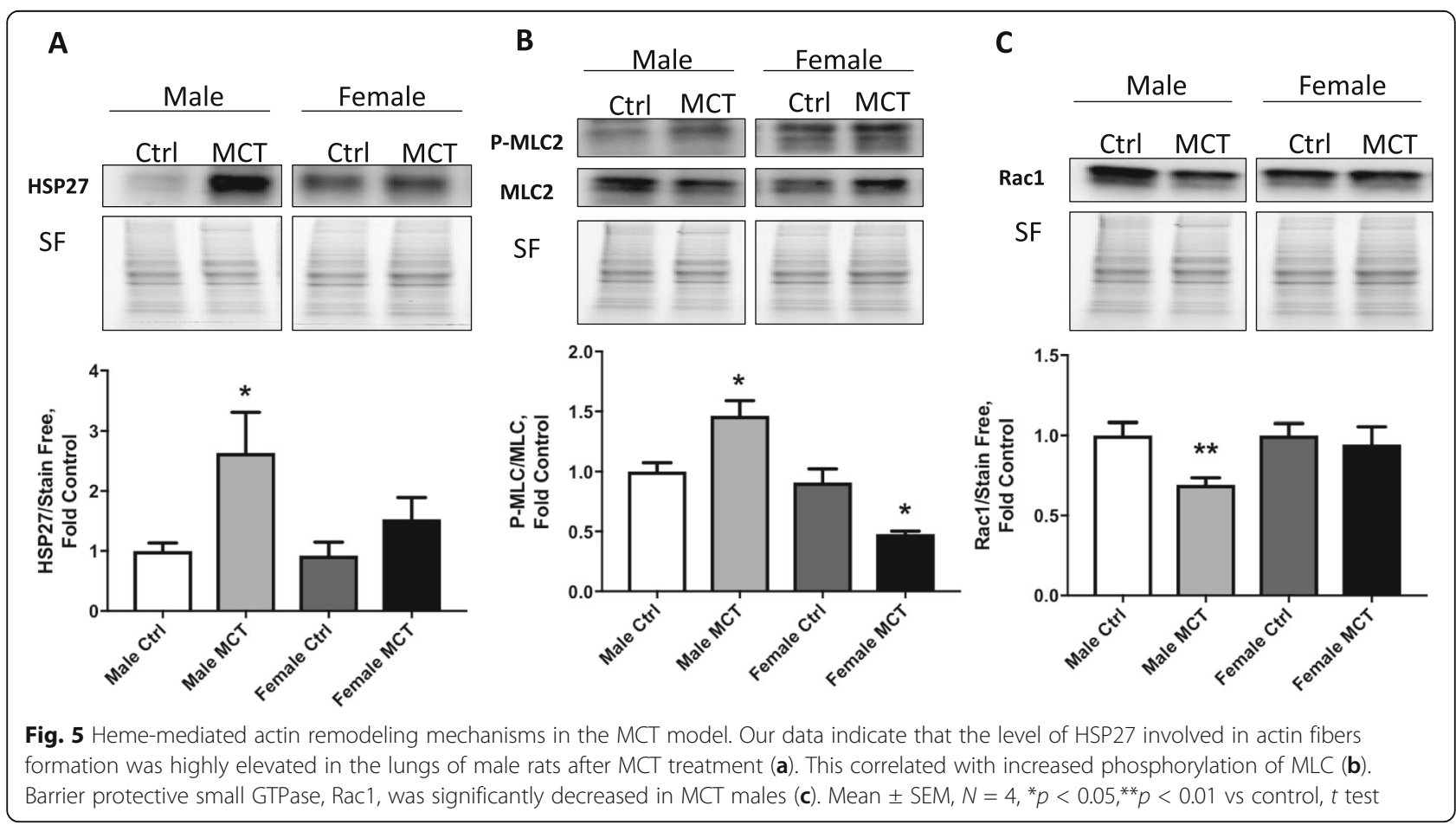




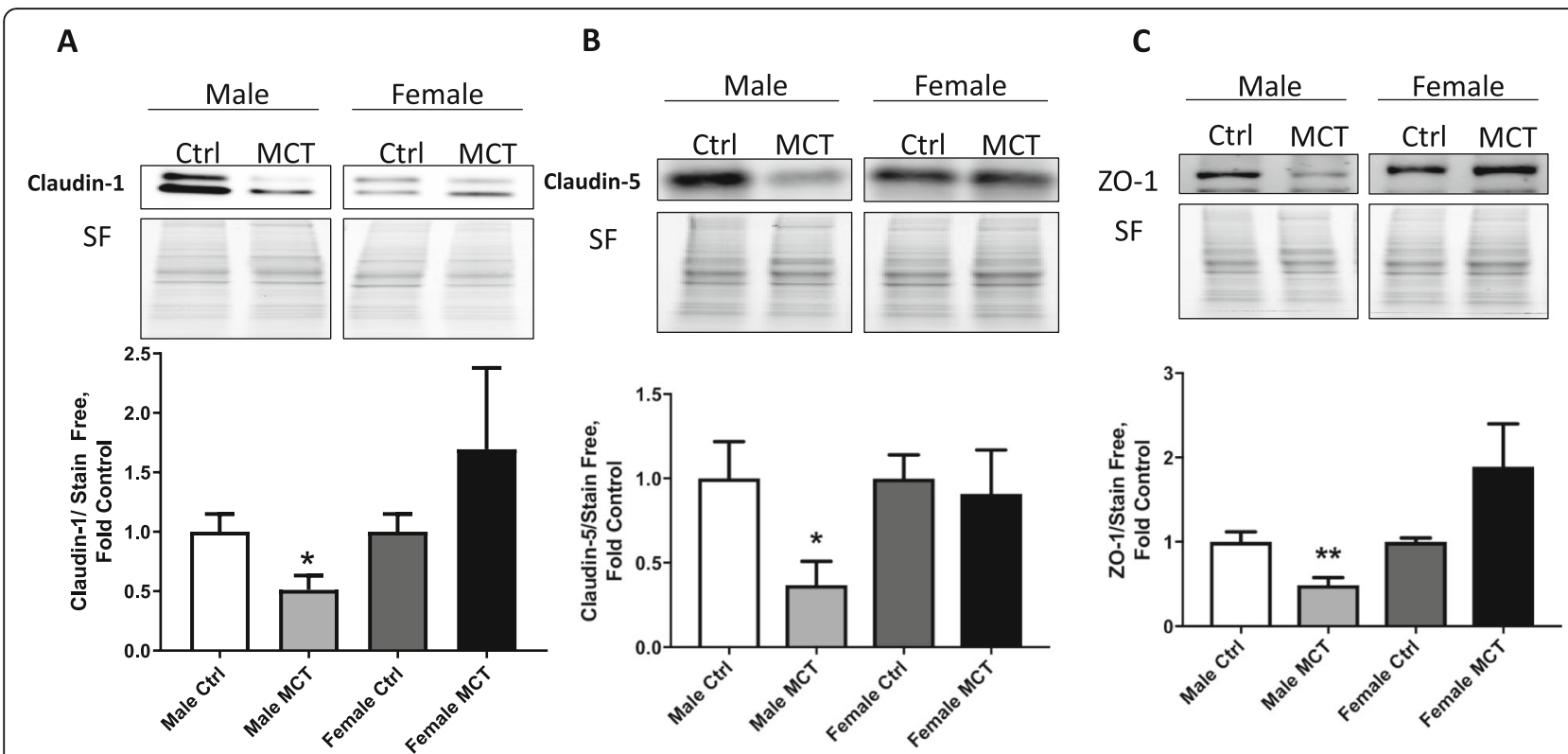

Fig. 6 Heme-mediated effects on tight junctions. Proteins involved in the formation of tight junctions, claudins1/5, and ZO-1 were significantly reduced in MCT male rats $(\mathbf{a}, \mathbf{b}, \mathbf{c})$. Mean $\pm \mathrm{SEM}, N=4,{ }^{*} p<0.05,{ }^{* *} p<0.01$ vs control, $t$ test

\section{Discussion}

It is well known that the frequency of $\mathrm{PH}$ in hemolytic patients is extremely higher than in the general population [22-28]. We have previously reported a strong correlation between increased hemolysis and the worsening of hemodynamic parameters in PH patients [17]. On the molecular level, we found that heme-mediated signaling can contribute to $\mathrm{PH}$ development via activation of p38 MAPK signaling. However, the role of hemolysis in $\mathrm{PH}$ is not entirely understood. In this work, we attempted to investigate the role of sex in hemolysis-induced effects. Sexual dimorphism in PH is a well-known phenomenon [29]. Although female sex associated with increased risk of $\mathrm{PH}$, males are having a severer disease and poorer prognosis. Our previous report in the Sugen/Hypoxia $(\mathrm{Su} / \mathrm{Hx})$ rat model showed that males develop inflammation and fibrosis in the lungs and heart, whereas females exhibited increased proliferation of pulmonary vasculature but adaptive right heart hypertrophy without fibrotic changes [30]. We also recently showed that rats with a mutation in NFU1 protein develop pulmonary arterial hypertension with a remarkable demonstration of sexual dimorphism as found in humans. Interestingly, in the NFU1 rat model, we found that the prevalence of the disease is more pronounced in female rats than in males [31]. In the present study, we found that the early MCT model indicates that males are more prone to develop hemolysis, and they showed increased pulmonary pressure and remodeling.

Next, in the MCT model, we also observed increased lung edema similar to previous reports in the $\mathrm{Su} / \mathrm{Hx}$ model [17]. Therefore, lung edema could be linked to a hemolytic event in males. Upregulation of heme carrier protein-1 (HCP-1) found in the lungs of males results in increased free heme uptake. Thus, activating of heme influx will result in intracellular heme signaling only in males. This was confirmed by the activation of heme catabolism via upregulated heme oxygenase. We have also found that heme induces disruptive barrier mechanisms leading to a reduction in tight junction (TJ) proteins ZO-1, and claudins1,5, which regulates fluid retention in the bloodstream by the endothelial barrier [32, 33]. At the same time, we observed an increased actin stressfibers formation signaling via HSP27 and Rac1 [34, 35]. All these events will ultimately lead to endothelial barrier leakage (Fig. 7). Importantly, those mechanisms were found to be upregulated in males only at the early stage of the disease.

One of the main questions of this study is how endothelial barrier dysfunction can contribute to the development of PH. Previously, we found that barrier dysfunction is pronounced during the early stages of pulmonary hypertension development [17]. Thus, this study was done on the 14 days MCT model, which is considered to be the initial stage. Leakage of pulmonary vasculature due to heme-mediated mechanisms results in fluid accumulation around the pulmonary arteries. This, in turn, can attract inflammatory cells and accumulate cytokines and chemokines. We have also shown a strong correlation between lung edema and pulmonary pressure, and the hypoxic environment of vessels surrounding by edema [2]. Moreover, this liquid layer will 


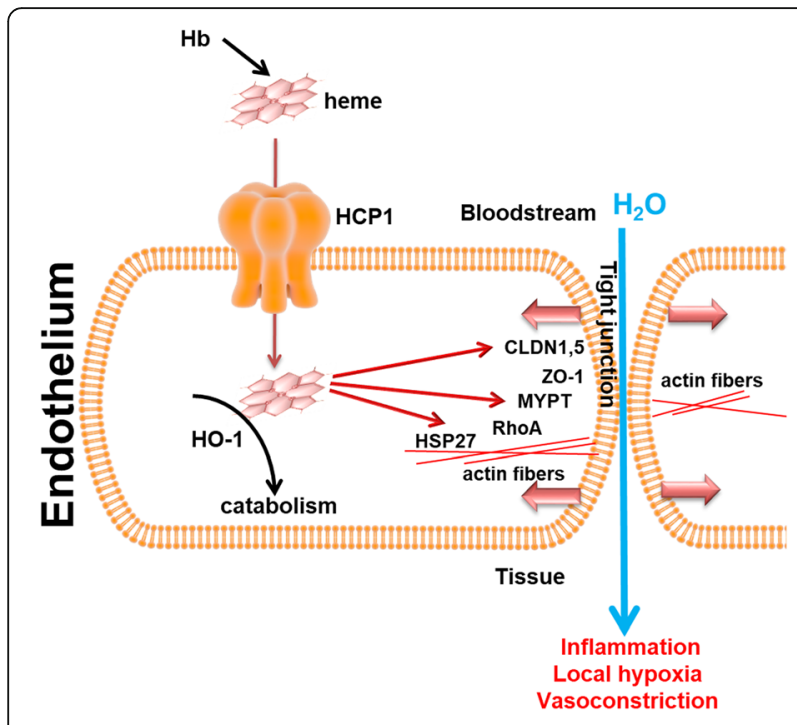

Fig. 7 Overall mechanisms of heme-induced barrier dysfunction. During hemolysis, hemoglobin releases its co-factor, free heme. Through the heme carrier protein 1 (HCP1), free heme translocates into cells, leading to the activation of heme oxygenase and barrier disruptive mechanisms. Heme affects the barrier in two phases. First, heme induces actin fibers formation by upregulation of HSP27, inactivation of barrier protective MYPT and Rac1, leading to increased phosphorylation of MLC. The second phase is the dysregulation of tight junctions $(\mathrm{TJ})$ between cells resulting in fluid leakage through the endothelial barrier. These effects of free heme contribute to the development of pulmonary hypertension via inflammation, vasoconstriction and local hypoxia due to the formation of perivascular cuffs

mechanically reduce the vasodilation of the arteries [36] and increase their stiffness. Finally, expansion of the perivascular area due to liquid accumulation will reduce gas and nutrients transport from vasa vasorum, leading to local ischemia. All those effects can induce initial damage to the pulmonary vasculature, induce hypoxia signaling leading to glycolytic switch and proliferation of smooth muscle cells and fibroblasts.

The predisposition of males to hemolysis and hemeactivated pathways could be explained by the wellknown observation that males have increased reactive oxygen species (ROS) generation, and the female hormone, estrogen, has an antioxidant action, protecting females [37]. Increased ROS generation was linked to the increased fragility of RBCs [38] that can contribute to elevated hemolysis in males. Moreover, our data indicate that males significantly upregulate heme oxygenase via NRF2 dependent mechanism. The role of heme oxygenase in $\mathrm{PH}$ is controversial [39-44]. On the one hand, heme oxygenase consumes the pro-oxidant, free heme, and produces an antioxidant, bilirubin. On another side, it releases carbon monoxide that can induce mitochondrial electron chain dysfunction and therefore contributes to a glycolytic switch. Moreover, heme oxygenase releases free iron, which is an essential oxidant involved in the Fenton reaction and is a cofactor of prolyl hydroxylases involved in the inactivation of HIF signaling. Thus, the overall vector of heme oxygenase action in $\mathrm{PH}$ is complex. Our data indicate that the activation of heme oxygenase in males is insufficient to stop hememediated signaling related to barrier disruptive mechanisms. Therefore, in spite of the activation of NFR2 and heme oxygenase, heme induces barrier leakage in the MCT model in male rats.

\section{Perspectives and significance}

We found that elevated hemolysis in males results in a more active translocation of free heme into the cells due to an upregulation of heme carrier ( $\mathrm{HCP}-1)$. Increased heme transport into the cell leads to the activation of heme oxygenase to catabolize intracellular free heme. Despite the heme oxygenase activation, intracellular free heme induces the multiple barrier-disruptive mechanisms via the reduction of tight junctions and activation of signaling proteins important for cytoskeleton rearrangement. The effects of free heme are illustrated in Fig. 7. This shows an important role of free heme signaling in barrier dysfunction at an early stage of PH. Therefore, treatments to reduce hemolysis and free heme should be applied for early-stage $\mathrm{PH}$ patients, patients with exercise-induced $\mathrm{PH}$, and patients with familial $\mathrm{PH}$, especially with the male sex.

\section{Authors' contributions}

RR, OR contributed to conception and design; CAE, MN, JJ, SK, MW contributed to analysis and interpretation; OR, JJ, RR, MN contributed to drafting the manuscript. All authors read and approved the final manuscript.

\section{Funding}

This work was supported by NHH grants R01HL133085 (OR) and R01HL132918 (RR)

Availability of data and materials

The datasets used and/or analyzed during the current study are available from the corresponding author on reasonable request.

Ethics approval and consent to participate

The handling procedures and experimental design were approved by the University of Arizona Institutional Animal Care and Use Committee.

Consent for publication

Not applicable.

Competing interests

The authors declare that they have no competing interests.

Received: 22 August 2019 Accepted: 10 March 2020

Published online: 18 March 2020

References

1. Batton KA, Austin CO, Bruno KA, Burger CD, Shapiro BP, Fairweather D. Sex differences in pulmonary arterial hypertension: role of infection and autoimmunity in the pathogenesis of disease. Biol Sex Differ. 2018;9(1):15.

2. Rafikova O, Al Ghouleh I, Rafikov R. Focus on Early events: pathogenesis of pulmonary arterial hypertension development. Antioxid Redox Signal. 2019.

3. Thenappan T, Ormiston ML, Ryan JJ, Archer SL. Pulmonary arterial hypertension: pathogenesis and clinical management. BMJ. 2018;360:55492. 
4. Ghouleh IA, Sahoo S, Meijles DN, Amaral JH, de Jesus DS, Sembrat J, Rojas M, Goncharov DA, Goncharova EA, Pagano PJ. Endothelial Nox1 oxidase assembly in human pulmonary arterial hypertension; driver of Gremlin1mediated proliferation. Clin Sci (Lond). 2017;131(15):2019-35.

5. Patel M, Predescu D, Tandon R, Bardita C, Pogoriler J, Bhorade S, Wang M, Comhair S, Hemnes AR, Chen J, et al. A novel p38 mitogen-activated protein kinase/Elk-1 transcription factor-dependent molecular mechanism underlying abnormal endothelial cell proliferation in plexogenic pulmonary arterial hypertension. J Biol Chem. 2013;288(36):25701-16.

6. Tsutsumi T, Nagaoka T, Yoshida T, Wang L, Kuriyama S, Suzuki Y, Nagata Y, Harada N, Kodama Y, Takahashi F, et al. Nintedanib ameliorates experimental pulmonary arterial hypertension via inhibition of endothelial mesenchymal transition and smooth muscle cell proliferation. PLoS One. 2019;14(7):e0214697.

7. Takahashi J, Orcholski M, Yuan K, de Jesus PV. PDGF-dependent betacatenin activation is associated with abnormal pulmonary artery smooth muscle cell proliferation in pulmonary arterial hypertension. FEBS Lett. 2016; 590(1):101-9.

8. Zurlo G, Piquereau J, Moulin M, Pires Da Silva J, Gressette M, Ranchoux B, Garnier A, Ventura-Clapier R, Fadel E, Humbert M, et al. Sirtuin 1 regulates pulmonary artery smooth muscle cell proliferation: role in pulmonary arterial hypertension. J Hypertens. 2018;36(5):1164-77.

9. Barman SA, Fulton D. Adventitial fibroblast Nox4 expression and ROS signaling in pulmonary arterial hypertension. Adv Exp Med Biol. 2017;967:1-11.

10. Marsboom G, Chen Z, Yuan Y, Zhang Y, Tiruppathi C, Loyd JE, Austin ED, Machado RF, Minshall RD, Rehman J, et al. Aberrant caveolin-1-mediated Smad signaling and proliferation identified by analysis of adenine 474 deletion mutation (c.474delA) in patient fibroblasts: a new perspective on the mechanism of pulmonary hypertension. Mol Biol Cell. 2017;28(9):1177-85.

11. Carrillo L, Aviado DM. Monocrotaline-induced pulmonary hypertension and p-chlorophenylalanine (PCPA). Lab Invest. 1969;20(3):243-8.

12. Hayashi $Y$, Lalich JJ. Renal and pulmonary alterations induced in rats by a single injection of monocrotaline. Proc Soc Exp Biol Med. 1967;124(2):392-6.

13. Sakao S, Taraseviciene-Stewart L, Lee JD, Wood K, Cool CD, Voelkel NF. Initial apoptosis is followed by increased proliferation of apoptosis-resistant endothelial cells. FASEB J. 2005;19(9):1178-80.

14. Taraseviciene-Stewart L, Kasahara Y, Alger L, Hirth P, Mc Mahon G, Waltenberger J, Voelkel NF, Tuder RM. Inhibition of the VEGF receptor 2 combined with chronic hypoxia causes cell death-dependent pulmonary endothelial cell proliferation and severe pulmonary hypertension. FASEB J. 2001;15(2):427-38

15. Rafikova O, Rafikov R, Kangath A, Qu N, Aggarwal S, Sharma S, Desai J, Fields T, Ludewig B, Yuan JX, et al. Redox regulation of epidermal growth factor receptor signaling during the development of pulmonary hypertension. Free Radic Biol Med. 2016;95:96-111.

16. Rafikova O, Meadows ML, Kinchen JM, Mohney RP, Maltepe E, Desai AA, Yuan JX, Garcia JG, Fineman JR, Rafikov R, et al. Metabolic changes precede the development of pulmonary hypertension in the monocrotaline exposed rat lung. PLoS One. 2016;11(3):e0150480.

17. Rafikova O, Williams ER, McBride ML, Zemskova M, Srivastava A, Nair V, Desai AA, Langlais PR, Zemskov E, Simon M, et al. Hemolysis-induced lung vascular leakage contributes to the development of pulmonary hypertension. Am J Respir Cell Mol Biol. 2018;59(3):334-45.

18. Schindelin J, Arganda-Carreras I, Frise E, Kaynig V, Longair M, Pietzsch T, Preibisch S, Rueden C, Saalfeld S, Schmid B, et al. Fiji: an opensource platform for biological-image analysis. Nature Methods. 2012; 9(7):676-82

19. Hira S, Tomita T, Matsui T, Igarashi K, Ikeda-Saito M. Bach1, a hemedependent transcription factor, reveals presence of multiple heme binding sites with distinct coordination structure. IUBMB Life. 2007:59(8-9):542-51.

20. Segawa K, Watanabe-Matsui M, Tsuda K, Matsui T, Shirouzu M, Igarashi K, Murayama K. Biophysical characterization of heme binding to the intrinsically disordered region of Bach1. Eur Biophys J. 2019;48(4):361-9.

21. Mammoto T, Parikh SM, Mammoto A, Gallagher D, Chan B, Mostoslavsky G, Ingber DE, Sukhatme VP. Angiopoietin-1 requires p190 RhoGAP to protect against vascular leakage in vivo. J Biol Chem. 2007;282(33):23910-8.

22. Al-Khoufi EA. Prevalence of pulmonary arterial hypertension among sickle cell disease patients in Al Hassa. Glob J Health Sci. 2013:5(5):174-80.

23. Amadi VN, Balogun MO, Akinola NO, Adebayo RA, Akintomide AO. Pulmonary hypertension in Nigerian adults with sickle cell anemia. Vasc Health Risk Manag. 2017;13:153-60.
24. Bigna JJ, Noubiap JJ, Nansseu JR, Aminde LN. Prevalence and etiologies of pulmonary hypertension in Africa: a systematic review and meta-analysis. BMC Pulm Med. 2017;17(1):183.

25. Fonseca G, Souza R. Pulmonary hypertension in sickle cell disease. Curr Opin Pulm Med. 2015;21(5):432-7.

26. Chueamuangphan N, Wongtheptien W, Nawarawong W, Sukornthasarn A, Chuncharunee S, Tawichasri C, Patumanond J. Clinical indicators for pulmonary arterial hypertension in thalassemia. J Med Assoc Thai. 2012;95(1):16-21.

27. Derchi G, Galanello R, Bina P, Cappellini MD, Piga A, Lai ME, Quarta A, Casu $G$, Perrotta S, Pinto $V$, et al. Prevalence and risk factors for pulmonary arterial hypertension in a large group of beta-thalassemia patients using right heart catheterization: a Webthal study. Circulation. 2014;129(3):338-45.

28. Moghaddam HM, Badiei Z, Eftekhari K, Shakeri R, Farhangi H. Prevalence of pulmonary hypertension in patients with thalassemia intermedia in 2009: a single center's experience. Electron Physician. 2015;7(3):1102-7.

29. Rafikov R, James J, McClain N, Tofovic SP, Rafikova O. Role of gender in regulation of redox homeostasis in pulmonary arterial hypertension. Antioxidants (Basel). 2019;8(5):135.

30. Rafikova O, Rafikov R, Meadows ML, Kangath A, Jonigk D, Black SM. The sexual dimorphism associated with pulmonary hypertension corresponds to a fibrotic phenotype. Pulm Circ. 2015;5(1):184-97.

31. Niihori M, Eccles CA, Kurdyukov S, Zemskova M, Varghese MV, Stepanova AA, Galkin A, Rafikov R, Rafikova O. Rats with human mutation of NFU1 develop pulmonary hypertension. Am J Respir Cell Mol Biol. 2019.

32. Bauer HC, Krizbai IA, Bauer H, Traweger A: "You Shall Not Pass"-tight junctions of the blood brain barrier. Front Neurosci 2014, 8:392.

33. Radeva MY, Waschke J. Mind the gap: mechanisms regulating the endothelial barrier. Acta Physiol (Oxf). 2018:222(1).

34. Wang W, Weng J, Yu L, Huang Q, Jiang Y, Guo X. Role of TLR4-p38 MAPKHsp27 signal pathway in LPS-induced pulmonary epithelial hyperpermeability. BMC Pulm Med. 2018;18(1):178.

35. Schnoor M, Garcia Ponce A, Vadillo E, Pelayo R, Rossaint J, Zarbock A. Actin dynamics in the regulation of endothelial barrier functions and neutrophil recruitment during endotoxemia and sepsis. Cell Mol Life Sci. 2017;74(11):1985-97.

36. Rafikova O, Srivastava A, Desai AA, Rafikov R, Tofovic SP. Recurrent inhibition of mitochondrial complex III induces chronic pulmonary vasoconstriction and glycolytic switch in the rat lung. Respir Res. 2018;19(1):69.

37. Vina J, Borras C, Gambini J, Sastre J, Pallardo FV: Why females live longer than males: control of longevity by sex hormones. Sci Aging Knowledge Environ 2005, 2005(23):pe17.

38. George A, Pushkaran S, Konstantinidis DG, Koochaki S, Malik P, Mohandas N, Zheng Y, Joiner CH, Kalfa TA. Erythrocyte NADPH oxidase activity modulated by Rac GTPases, PKC, and plasma cytokines contributes to oxidative stress in sickle cell disease. Blood. 2013;121(11):2099-107.

39. Christou H, Morita T, Hsieh CM, Koike H, Arkonac B, Perrella MA, Kourembanas S. Prevention of hypoxia-induced pulmonary hypertension by enhancement of endogenous heme oxygenase-1 in the rat. Circ Res. 2000; 86(12):1224-9.

40. Hsu HH, Ko WJ, Hsu JY, Chen JS, Lee YC, Lai IR, Chen CF. Simvastatin ameliorates established pulmonary hypertension through a heme oxygenase-1 dependent pathway in rats. Respir Res. 2009;10:32.

41. Iwasaki T, Takahashi T, Shimizu H, Ohmori E, Morimoto T, Kajiya M, Takeuchi M, Morita K, Akagi R, Kajiya F. Increased pulmonary heme oxygenase-1 and delta-aminolevulinate synthase expression in monocrotaline-induced pulmonary hypertension. Curr Neurovasc Res. 2005;2(2):133-9.

42. van Loon RL, Bartelds B, Wagener FA, Affara N, Mohaupt S, Wijnberg H, Pennings SW, Takens J, Berger RM. Erythropoietin attenuates pulmonary vascular remodeling in experimental pulmonary arterial hypertension through interplay between endothelial progenitor cells and heme oxygenase. Front Pediatr. 2015;3:71.

43. Zhang $W H$, Zhang YJ, Liu CP, Yu BX, Lu WX. Simvastatin protects against the development of monocrotaline-induced pulmonary hypertension in rats via a heme oxygenase-1-dependent pathway. Exp Lung Res. 2011;37(8):492-9.

44. Zhou H, Liu H, Porvasnik SL, Terada N, Agarwal A, Cheng Y, Visner GA. Heme oxygenase-1 mediates the protective effects of rapamycin in monocrotalineinduced pulmonary hypertension. Lab Invest. 2006;86(1):62-71.

\section{Publisher's Note}

Springer Nature remains neutral with regard to jurisdictional claims in published maps and institutional affiliations. 